\title{
PROVIDER-INITIATED COUNSELLING AND TESTING (PICT): AN OVERVIEW
}

\author{
Nondumiso Makhunga-Ramfolo ${ }^{1}, M B C h B, M S c$ (Clin Epidemiol) \\ Thato Chidarikire ${ }^{2}, M S c$ (Med) \\ Thato Farirai $^{\mathbf{3}}, B S W$, Hon $S o c$ Sci \\ Refiloe Matji ${ }^{1}, M D, M P H, D T C D$ \\ ${ }^{1}$ University Research Co., LLC (URC), Pretoria \\ ${ }^{2}$ HIV and AIDS and STIS Cluster, National Department of Health, Pretoria \\ ${ }^{3}$ Centers for Disease Control and Prevention (CDC-SA), Pretoria
}

South Africa has the highest number of people living with HIV in the world. Despite this, many South Africans do not know their HIV status and uptake of voluntary counselling and testing (VCT) has been suboptimal. In clinical settings there are many missed opportunities for HIV diagnosis as most patients are not routinely offered HIV counselling and testing (HCT). Provider-initiated counselling and testing (PICT) has been introduced to ensure that HCT becomes the standard of care in all consultations with health providers. PICT promotes universal access to prevention, care and treatment services for all clients by increasing the utilisation and acceptance of HCT services.

This article outlines the rationale for PICT as well as providing an overview of the implementation protocol that will equip health care providers with the knowledge required to integrate HCT into routine medical care.

\section{THE EPIDEMIOLOGY OF HIV}

According to the World Health Organization (WHO), in 2007 more than 33 million people were living with HIV/AIDS with at least 2.7 million new infections being transmitted annually. ${ }^{1}$ In a mid-term review of the National Strategic Plan 2007 - 2011, the Human Sciences Research Council reported that while the HIV epidemic in South Africa appears to have stabilised, a significant number of South Africans do not know their HIV status and testing is still primarily client initiated. ${ }^{2}$ According to the South African National HIV Prevalence, Incidence, Behaviour and Communication Survey, 2008, South Africa has an estimated national HIV prevalence of $10.6 \%$ (5.3 million people). Despite the availability of voluntary counselling and testing (VCT) services since 2000, many South Africans still do not know their HIV status. HIV-infected patients who consult their family practitioners are still being missed as opportunities to test are lost.

In 2007, the WHO made recommendations to introduce provider-initiated counselling and testing (PICT) in addition to client-initiated counselling and testing, also known as VCT, as an effective public health intervention to increase access to HIV counselling and testing (HCT) and reduce missed opportunities for testing. ${ }^{3}$ With PICT the health care provider routinely offers and recommends an HIV test to all clients, irrespective of the medical diagnosis. The main objectives are to integrate HIV testing into routine medical care, thereby facilitating early diagnosis. By implementing PICT, family practitioners can not only learn the client's HIV status, allowing for appropriate clinical decisions to be made, but also enable all clients to know their status. Early diagnosis improves health outcomes of those who are HIV positive, while ensuring that they are provided with information to reduce transmission.

The recent HCT policy guidelines from the National Department of Health $(\mathrm{NDoH})^{4}$ emphasised the need to complement VCT through the implementation of PICT by all health care providers in both the public and private sectors. The overall goal of this strategy is to assist health care providers to expand access to HCT for their clients, thereby reducing the burden of disease in communities.

\section{PICT AS A GATEWAY TO HIV PREVENTION,}

CARE, TREATMENT AND SUPPORT SERVICES

The availability of HIV rapid tests and same-day results has increased access to accurate, reliable and costeffective diagnosis. HIV rapid tests allow medical practitioners to test their clients and provide results within a short space of time. The relationship between medical practitioners and their patients places them in an ideal situation to offer patient-centred care, allowing for better decisions to be made. For patients visiting medical practitioners, PICT is an important and effective model that forms part of the broader prevention strategy and acts as the gateway to accessing care, support and treatment services. 


\section{BEYOND VCT - DIFFERENCES BETWEEN \\ PICT AND VCT}

While there are many similarities between PICT and VCT, it is important for the medical practitioner to understand the differences between the two models (Table I).

\section{SIMILARITIES BETWEEN PICT AND VCT}

Both VCT and PICT are voluntary and require consent from the client. In both models testing is always performed in the client's best interests, in keeping with acceptable principles of medical ethics, and HIV results are always reported back to the client. In both models the client is supported to deal with the HIV test results. Counselling always precedes and follows testing.

\section{BENEFITS OF PICT}

Knowing the client's HIV status can have benefits for the individual concerned, the provider and the community. For HIV-negative people, knowing their status empowers them to protect themselves from becoming infected with HIV. It provides them with information on how to remain negative by assessing their own behaviour and providing solutions for behaviour change. For HIV-positive people, knowing their status ensures that they can be provided with the appropriate treatment, care and support services and assists them in living positively. Couples who know their HIV status are empowered to make safer choices with respect to sexual behaviour, e.g. condom use in discordant couples, implementation of positive living strategies, and accessing treatment for the prevention of mother-to-child transmission (PMTCT) of HIV.

PICT enables medical practitioners to treat their clients appropriately by identifying those who need treatment and/or wellness programmes early. This helps health care providers to improve the quality of medical care rendered to their clients and reduce morbidity and mortality.

PICT assists in reducing stigma in the community by making HIV testing the norm. It leads to the expansion of care and support services to deal with the demand for services.

\section{PRINCIPLES OF PICT}

PICT does not imply that people are coerced to test, nor does it constitute compulsory or mandatory testing.
In implementing PICT medical practitioners should be guided by three principles, viz. consent, counselling and confidentiality, also known as the three Cs. Inappropriate use of PICT diminishes trust in health care providers and can lead to poor adherence to treatment and inadequate uptake of referrals.

\section{INFORMED CONSENT}

HIV testing by medical practitioners should only occur when the client or his or her legal surrogate, e.g. parent or guardian, has provided informed consent. The client must be provided with information that is understandable according to his or her language, disability and literacy. The client must also understand the nature of the test and its consequences and also understand the purpose of the exchange of information as being in the best interests of his or her own health, that of the partner, and in the case of a pregnant woman, the fetus or the infant being breastfed.

\section{The PICT protocol}

Implementing PICT in the medical practitioner's rooms has specific steps that need to be followed. The PICT protocol is set out in Fig. 1.

\section{Health education}

Education is aimed at providing basic information to clients on HIV and the PICT process. Education can be provided to an individual verbally and can be supported by other material, e.g. pamphlets and audiovisual tools. The client's right to refuse to be tested should be discussed. The content of health education should cover the following:

- the difference between HIV and AIDS

- how HIV is acquired and transmission

- HIV prevention measures and options for prevention, e.g. medical male circumcision, prevention with positives to prevent transmission to HIV negative partners, reduction in the number of concurrent sexual partners, correct regular condom use and PMTCT

- the advantages of testing and the importance of early diagnosis

- assurance that the process is confidential and of the right to privacy, and that only those directly involved in the person's care will be informed about their HIV status

\section{TABLE I. DIFFERENCES BETWEEN PICT AND VCT}

\section{PICT}

Individual is seeking medical care and HCT is recommended and performed by medical practitioner as part of the consultation

Services provided are confidential and documented in medical record to ensure continuity of care

Primary focus is on identifying HIV-infected people and linking them with prevention, care and treatment services

Verbal consent is required and should be documented in the patient record

First user of the test result is the health care worker to make a correct diagnosis and provide appropriate treatment

\section{VCT}

Individual chooses to seek HCT

Anonymous or confidential services may be offered

Primary focus is on preventing HIV acquisition through risk assessment, risk reduction and testing

Written consent or thumb print for illiterate clients is required

First user of the test result is the client, who uses the information to make personal life decisions 


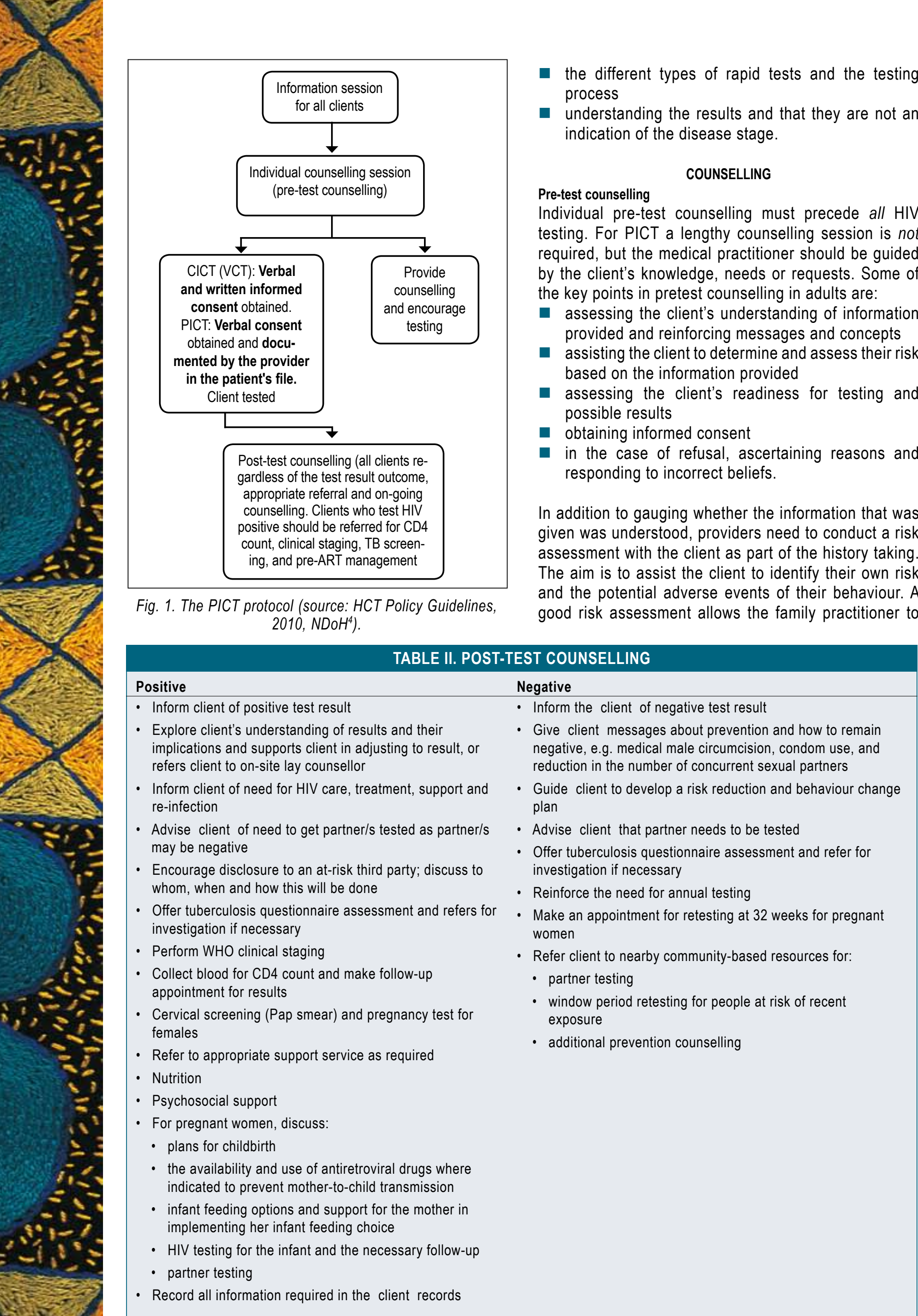


devise an individual risk reduction plan with the client. A risk assessment can be incorporated in the history taking and systemic enquiry about: ${ }^{5}$

- alcohol use

- drug use (especially intravenous drug use)

- domestic violence

- history of prison incarceration

- sexual history, including:

- number of previous and current partners

- history of unprotected high-risk sexual intercourse, anal and vaginal

- rape or sexual assault

- sexually transmitted infections.

\section{Post-test counselling}

All clients who have been tested should receive post-test counselling (Table II), irrespective of HIV results. The content of post-test counselling will be guided by the HIV test results.

\section{Testing}

HIV rapid tests are easy to perform with proper training. Results can be provided within 10 - 15 minutes during client consultation and are as reliable and accurate as enzyme immunoassays (EIA). All persons performing HIV rapid testing should follow a stipulated quality assurance programme to ensure accurate and reliable results. HIV testing should be conducted using the accepted national HIV testing algorithm using both a screening and a confirmatory test, as indicated in Fig. 2.

\section{CONFIDENTIALITY AND DISCLOSURE}

While HCT is a confidential process, clients should be encouraged to disclose their results to their sexual partners. The concept of shared confidentiality, i.e. that health care providers who contribute directly to the care of the person may have access to his or her results, should be discussed with the client. Medical practitioners may also offer to help clients to disclose to their partners. Medical practitioners should be cautioned against disclosing HIV results to third parties without either the client's written consent or a court order. Where any doubt exists about the appropriate course of action, medical practitioners should consult with senior colleagues for guidance.

\section{Acknowledgements}

This article was supported by Cooperative Agreement number U62/PS325199-05 from the Centers for Disease Control and Prevention (CDC). Its contents are solely the responsibility of the authors and do not necessarily represent the official views of CDC.

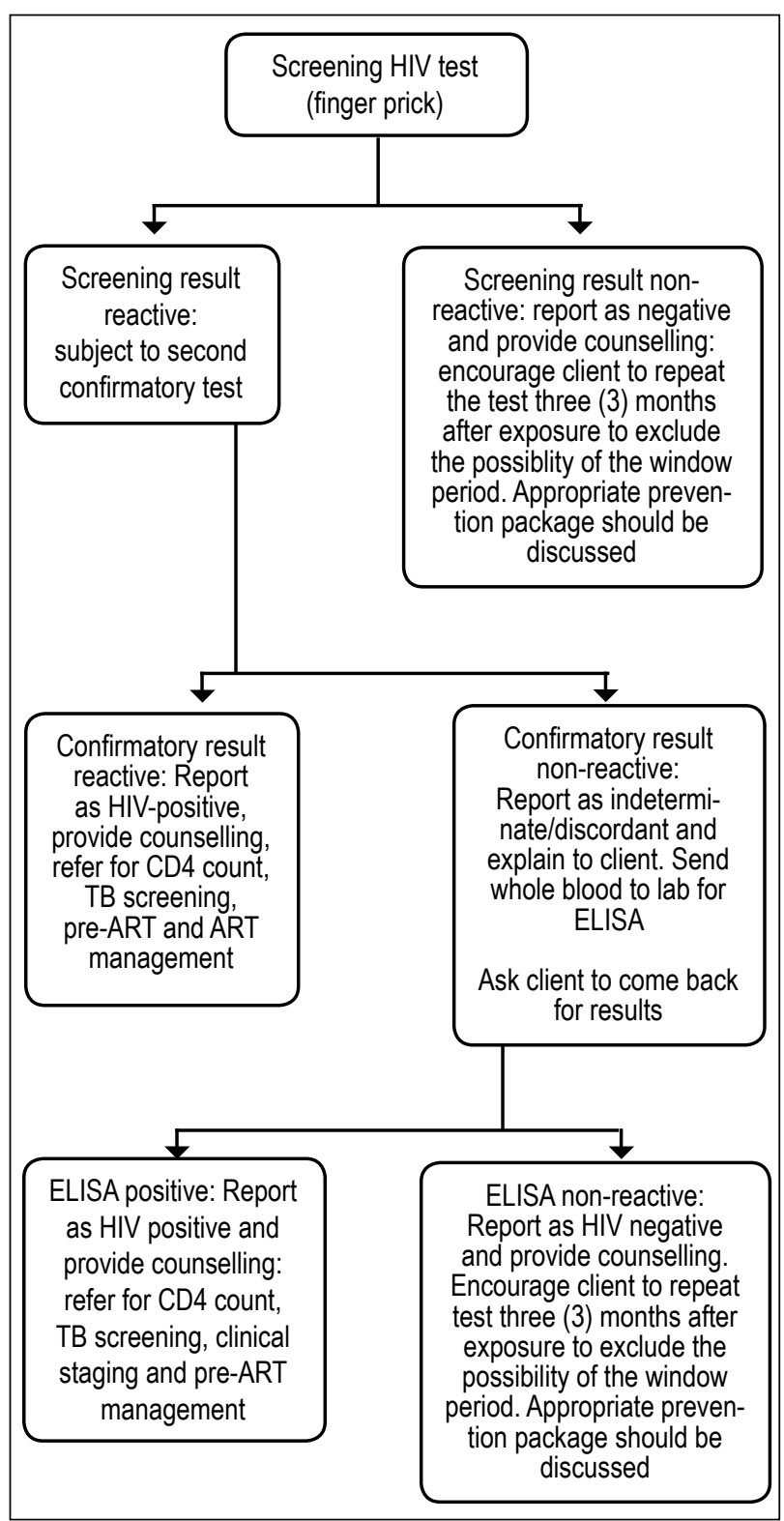

Fig. 2. National HIV testing algorithm (source: HCT Policy Guidelines, 2010, $\mathrm{NDoH}^{4}$ ).

\section{REFERENCES}

1. World Health Organization/UNAIDS. Guidance on provider-initiated HIV testing and counselling in health facilities. May 2007. http://whqlibdoc.who.int/ publications/2007/9789241595568_eng.pdf (accessed 15 April 2010).

2. Shisana O, Rehle T, Simbayi LC, et al., and the SABSSM III Implementation Team. South African National HIV Prevalence, Incidence, Behaviour and Communication Survey 2008: A Turning Tide among Teenagers? Cape Town: HSRC Press, 2009.

3. World Health Organization/UNAIDS. Guidance on Provider-initiated HIV Testing and Counselling in Health Facilities, May 2007. Geneva: WHO, 2007.

4. National Department of Health. HCT Policy Guidelines March 2010. Pretoria: NDoH, 2010.

5. Coates T, Mayer K, Makadon H, Schechtel J. HIV risk assessment: physician and patient communication. J Gen Intern Med 1997;12(11):722-723. 